\title{
Chronic ulcers: considerations about contaminant flora, infection and antimicrobial treatment ${ }^{*}$
}

\author{
Luciana Patrícia Fernandes Abbade ${ }^{1}$
}

Hélio Amante Miot ${ }^{1}$

Mr. Editor,

Although Hansen's disease epidemic still reaches important numbers in Brazil and a significant portion of patients are diagnosed with disabilities already installed, the study of leprosy ulcers is a priority in public health.

Chronic ulcers greatly impact the quality of life of patients, require time and specific care for complete healing, burdening the health and social security systems. Besides the influence of clinical comorbidities and leprosis neuropathy, critical colonization, episodes of cutaneous or soft parts infection and osteomielitis are complications of the healing process which justify a meticulous preliminary clinical investigation for its identification and early treatment. ${ }^{1}$

After careful reading of the excellent text of Gelatti et $\mathrm{al}^{2}{ }^{2}$ which explored microbiological aspects of chronic ulcer exudation in individuals cured of leprosy, some questions of clinical care aspect arise that we believe deserve consideration.

In this study, ${ }^{3}$ the authors did not mention topography, dimension, time of evolution and clinical characteristics of ulcers, such as: infection, coloniza- tion, necrosis / devitalized tissue, previous or current forms of treatment. Such elements may interfere both quantitatively and qualitatively with the bacterial flora found and treatment strategy. ${ }^{3}$

The technique for collection of ulcer exudation from the deeper part using a sterile swab, even after careful cleansing does not guarantee that the cultures represent anything more than the contaminating flora, with no predictability for agents that may trigger infection of the skin, soft parts or osteomielitis, as well as guide the antibiotic therapy.

Even though swabs are contraindicated by experts, ${ }^{4}$ they are still routinely used in basic and hospital care as an antimicrobial strategy guide in case of chronic ulcers, oftentimes only colonized, without indication for systemic antibiotic treatment. A broad spectrum topical or systemic antibiotic therapy contributes to the selection of resistant strains in the entire body, imparting adverse effects and costs to the health system, in addition to failing to associate with higher healing rates, if there is no patent infection. ${ }^{5}$

The diagnosis of cutaneous or soft parts infec-

\footnotetext{
Work submitted on 09.07.2014

Approved by the Advisory Board and accepted for publication on 10.07.2014.

* Letter prepared by Department of Dermatology and Radiotherapy of Universidade Estadual Paulista "Júlio de Mesquita Filho" (Unesp) - Botucatu (SP), Brazil. Conflict of interest: None

Financial funding: None

Universidade Estadual Paulista “Júlio de Mesquita Filho” (Unesp) - Botucatu (SP), Brazil.
} 
tion should be based on clinical findings like erythema, edema, pain, local heat, alteration of the exudation pattern and devitalized tissue. It needs to be treated with empirical systemic antibiotic therapy in case a fragment of the ulcer bed cannot be qualitatively and quantitatively cultivated.

Likewise, the osteomielitis diagnosis should be based on clinical criteria and imaging exams such as $\mathrm{X}$ ray (chronic lesions), magnetic resonance and scintigraphy; cases should be referred to a reference service for care by an orthopedist. The culture of a bone frag- ment may guide the prescribed antibiotic therapy.

Our considerations do not discredit the results of the article of Gelatti and colleagues,; ${ }^{2}$ however, they alert to the implications of dermatological preliminary investigation in the evaluation of critical colonization and infection of chronic ulcers in basic care. Furthermore, they emphasize the fact that indication and orientation of systemic antibiotic therapy should not be based on results of exudation cultures, since adequate clinical and laboratorial correlation is required.

\section{REFERENCES}

1. Scotton MF, Miot HA, Abbade LP. Factors that influence healing of chronic venous leg ulcers: a retrospective cohort. An Bras Dermatol. 2014;89:414-22.

2. Gelatti LC, Bonamigo RR, Becker AP, Eidt LM, Ganassini L, D' Azevedo PA. Phenotypic, molecular and antimicrobial susceptibility assessment in isolates from chronic ulcers of cured leprosy patients: a case study in Southern Brazil. An Bras Dermatol. 2014;89:404-8.

3. de Souza JM, Vieira EC, Cortez TM, Mondelli AL, Miot HA, Abbade LP. Clinical and microbiologic evaluation of chronic leg ulcers: a cross-sectional study. Adv Skin Wound Care. 2014;27:222-7.

4. Bonham PA. Swab cultures for diagnosing wound infections: a literature review and clinical guideline. J Wound Ostomy Continence Nurs. 2009;36:389-95.

5. O'Meara S, Al-Kurdi D, Ologun Y, Ovington LG, Martyn-St James M, Richardson R. Antibiotics and antiseptics for venous leg ulcers. Cochrane Database Syst Rev 2014;1:CD003557.

MAILING ADDRESS:

E-mail: lfabbade@fmb.unesp.br

E-mail: heliomiot@fmb.unesp.br 